\title{
Is there any role of intermittent fasting in the prevention and improving clinical outcomes of COVID-19?: intersection between inflammation, mTOR pathway, autophagy and calorie restriction
}

\author{
Martin Gnoni $^{1,2} \cdot$ Renato Beas $^{3}$ (D) Raúl Vásquez-Garagatti $^{4,5,6}$
}

Received: 1 March 2021 / Accepted: 20 May 2021 / Published online: 4 June 2021

(C) Indian Virological Society 2021

\begin{abstract}
The coronavirus disease 2019 (COVID-19) pandemic is provoking a global public health crisis. Even though the academic world is intensively pursuing new therapies, there is still no "game changer" in the management of COVID 19. The Mammalian Target of Rapamycin (mTOR) is an ancient signaling system that has been proposed as a molecular tool used by coronaviruses and other RNA and DNA viruses in order to replicate and persist in the host cell. In recent years, Intermittent Fasting (IF), a practice consisting on a strict calorie restriction during a prolonged period of time during the day, has gained popularity due to its potential benefits in multiple health systems and in regulating inflammation. IF inhibits the mTOR pathway which is similar to the effects of Rapamycin in some animal models. mTOR inhibition and promotion of autophagy could potentially be the link between the possible direct benefits of IF in COVID-19 due to the interruption of the viral cycle (protein synthesis). Besides, IF has shown to be a strong anti-inflammatory in multiple prior studies, and may play a role in attenuating
\end{abstract}

Renato Beas

renatobeas@gmail.com

1 Department of Internal Medicine, Good Samaritan Hospital, Cincinnati, OH, USA

2 Division of Infectious Diseases, Department of Medicine, University of Louisville Health Sciences Center, Louisville, KY, USA

3 School of Medicine, Universidad Peruana de Ciencias Aplicadas, Lima, Peru

4 University of Tennessee Medical Center, Knoxville, TN, USA

5 Cherokee Health, Knoxville, TN, USA

6 Fruitstreet Health, New York, NY, USA
COVID -19 severity. This review hypothesizes the possible intersection between viral, immunological, and metabolic pathways related to mTOR and the potential mechanisms through which IF may improve clinical outcomes. Future prospective randomized controlled clinical trials to evaluate intermittent fasting (IF) regimens in order to prevent and treat moderate to severe forms of COVID-19 in humans are needed.

Keywords COVID-19 · Pandemic · Intermittent fasting · mTOR

\section{Introduction}

We are in the midst of a pandemic due to SARS-CoV-2 (named by WHO on Feb 11, 2020) that causes coronavirus disease 2019 (COVID-19) and is causing a global public health crisis [48]. Innovative therapies are in the lookup around the world in international academic centers while vaccines are starting to be distributed around the globe [37]. To this day, COVID-19 management is still continuously changing. The antiviral Remdesivir has been authorized for the treatment of suspected or laboratoryconfirmed COVID-19 in hospitalized patients with severe disease after data showed a decrease in the time to improvement from 14 to 11 days without clear benefit in mortality [51]. Dexamethasone also showed promising results when it proved to reduce mortality among those receiving invasive mechanical ventilation or oxygen at randomization [47]. Other therapeutics are being studied currently in the United States and worldwide which can be found elsewhere [51]. Even though the academic world is intensively researching this topic, there is still no "game changer" in the management of COVID 19. 
Recently, the molecular mechanisms that allow the SARS-CoV-2 and other Human Coronavirus to fully display its pathogenic capacity have been under scrutiny $[7,21]$. The Mammalian Target of Rapamycin (mTOR) is an ancient signaling system that has been proposed as a molecular tool used by this virus and other RNA and DNA viruses in order to replicate and persist in the host cell [7, 12, 50, 52, 61, 69]. In recent years, Intermittent Fasting (IF), a practice consisting on prolonged calorie restriction during the day with short feeding windows (also called, Time Restrictive Feeding-TRF), has gained popularity due to its potential benefits in multiple health systems and in regulating inflammation [14, 44].

Therefore, in this review we will be discussing and exploring the molecular mechanisms through which Intermittent Fasting (IF) may switch off the mTOR pathway and interfere with the SARS-CoV-2 cell replication cycle. This could attain important consequences not only for prevention but improving clinical outcomes in COVID-19 patients, especially in high-risk populations.

\section{The mammalian target of rapamycin (mTOR), cell growth, and survival}

The Mammalian Target of Rapamycin (mTOR), a serinethreonine kinase, is a complex molecular compound that integrates the input from multiple upstream pathways. It's a highly conserved ancient complex that senses cell nutrition, oxygen, stress, hormonal levels, and energy balance. It regulates metabolism, immunity, and physiology and is regulated by growth factors, hormones, cytokines, stress, and nutrients. Importantly, mTOR hyperactivation is highly linked to "aging" through "inhibition of autophagy" and accumulation of cellular debrides. Hyperactivation of mTOR has been linked to cardiovascular disease, inflammatory conditions, autoimmune diseases, aging, and cancer. Since SARS-CoV-2 triggers a "Cytokine stormlike Syndrome" (CSS) and because mTOR is linked to inflammatory conditions, this intriguing system has raised a lot of interest as a potential therapeutic target against COVID-19 [46, 55, 68]. More recently, there are multiple ongoing clinical trials using mTOR inhibitors like Rapamycin against SARS-CoV-2 (see below) [11]. One of the mechanisms for cell growth triggered by mTOR is the 5'cap-dependent mRNA translation by the assembled eIF4F complex responsible for mRNA translation into proteins [7]. The Cap-dependent mRNA translation is regulated by mTORC1 via the phosphorylation of downstream effectors. Briefly, the chain of events starts with the activation of PI3K (through a transmembrane ligand receptor) which is responsible for the phosphorylation of PIP-2 to PIP-3 (2nd messenger). PIP-3 then will phosphorylate Akt, which through phosphorylation of TSC2 will promote the formation of Rheb-GTP and final activation of mTOR 1 at the level of the lysosomal membrane (see Fig. 1). The above events will finalize with the activation of the eukaryotic initiation factor $4 \mathrm{E}$ (eIF4E)-binding protein 1 (4E-BP1) and the p70 ribosomal S6 kinase 1 (S6K1) resulting in protein translation [29] (Fig. 1). As it is well known, protein synthesis utilizing the cell machinery is of paramount importance on the life cycle of multiple family of virus including Coronaviruses. The mTOR complex may sense the "need for cell growth" in the presence of upstream stimulators and will start the protein translation process as described above (5'cap-dependent mRNA translation).

\section{mTOR, protein synthesis and infections}

It is not surprising that multiple pathogens naturally selected this cell machinery apparatus to replicate intracellularly and persist not only in chronic-latent infections but in acute infections as well. In particular this may be important for small RNA (+) virus since the inhibition of apoptosis and promotion of protein synthesis should play an important role to complete their life cycle within the cell before it perishes due to apoptosis or elimination within the phagolysosomes, like shown on RSV [12]. A recent study showed that Erlichia can activate mTOR through the MyD88 pathway activating the inflammasome in macrophages [26]. Interestingly, the same study showed that MyD88 knock-out mice did not cause inflammasome activation which implies that the MyD88 is actively involved in mTOR activation. It was also shown that other RNA and DNA viruses utilize the mTOR pathway of the host cell to self-replicate or avoid specific immune responses, "hijacking" the system [29]. Previous studies also confirmed the mTORC1 as the key factor in multiple virus life cycles, including hantavirus, hepatitis $\mathrm{C}$ virus, and coronaviruses [52, 61, 69]. Multiple bacterial species and parasites utilize different strategies, some of them inhibiting or activating the system. For example, Toxoplasma gondii activates mTORC1 to increase the translation of mTOR-sensitive transcripts, including those encoding proteins for mitochondrial function [43].

\section{mTOR and the coronavirus family of viruses}

Single-strand positive sense RNA viruses (SS-RNA+) like SARS-CoV-2 may use this system on its own benefit in order to increase the synthesis of viral proteins while inhibiting autophagy, at least transiently. Even though it remains uncertain on how SARS-CoV-2 may activate the mTOR pathway we hypothesize that it may be through the Toll Like Receptor (TLR) 7 or 9 activation after the initial processing of the virion at the post-entry-phago-lysosomal 


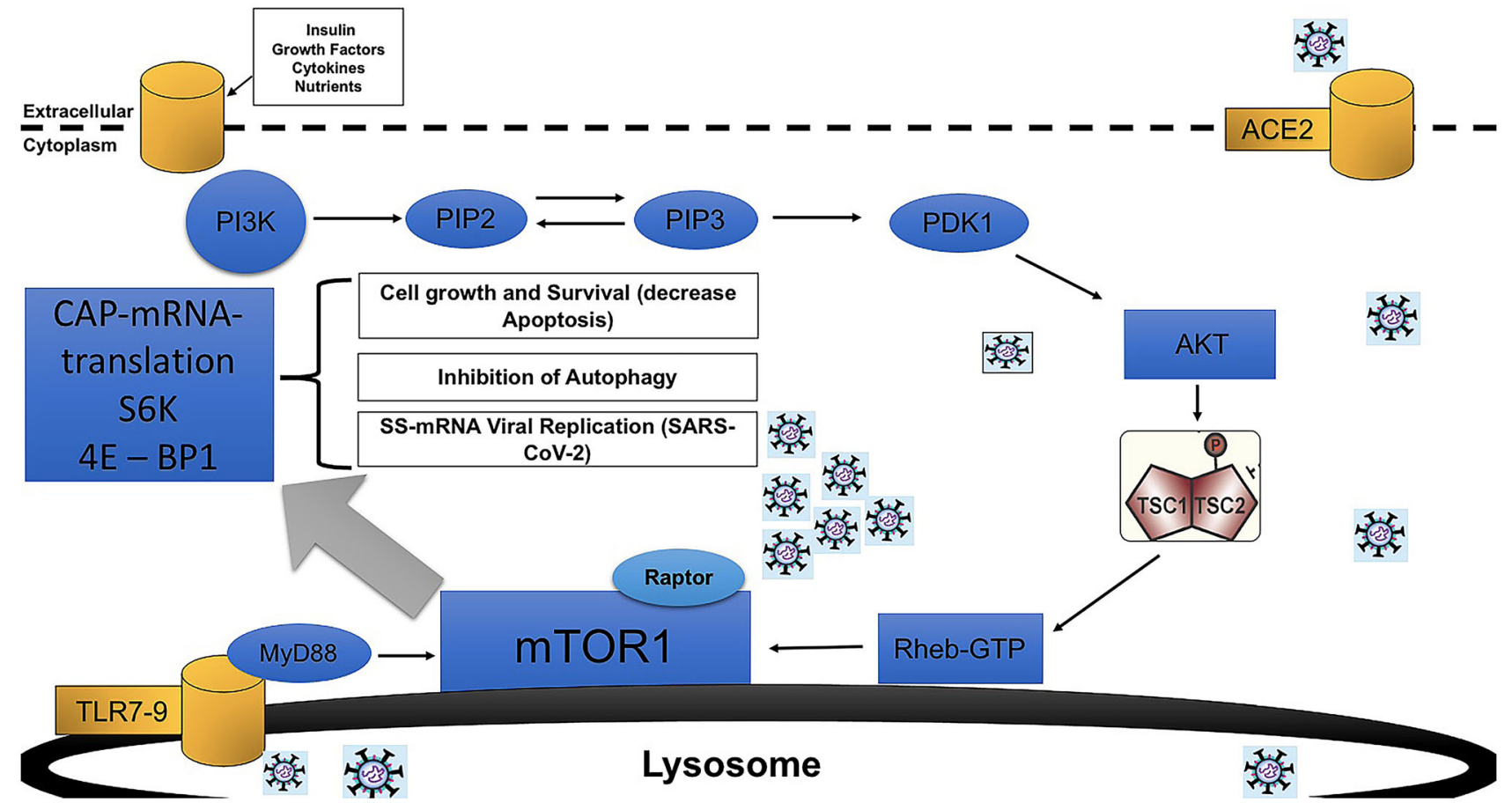

Fig. 1 Mechanisms of activation of the eukaryotic initiation factor $4 \mathrm{E}$ (eIF4E)-binding protein 1 (4E-BP1) and the p70 ribosomal S6 kinase 1 (S6K) resulting in protein translation leading to Cell growth and Survival, Inhibition of Autophagy and Viral Replication. The process starts with the activation of PI3K (through a transmembrane ligand receptor) that phosphorylates PIP-2, which through subsequent phosphorylation of TSC2 will promote the formation of Rheb-GTP

level. In a "fed-state" with availability of nutrients or other hormonal signals, the mTOR pathway may be activated by both, a nutrient-hormonal pathway and by a SARS-CoV-2TLR7/9-MyD88-dependent pathway (see Fig. 2).

In other members of the Human Coronavirus Family of Virus $(\mathrm{HCoV})$ it has been shown that the cap-dependent mRNA translation may, in fact, play an important role for the replication and survival of this family of viruses. In a recent study of the Middle East Respiratory Syndrome (MERS) the inhibition of mTOR with sirolimus or everolimus (mTOR inhibitors) reduced MERS-CoV infection by $60 \%$ implying a critical role for mTOR in the MERS$\mathrm{CoV}$ viral cycle. Of note, MERS-CoV shares many similarities with SARS-CoV-2 [55]. Another in vitro study done in 2015 showed that the ERK/MAPK and PI3K/AKT/ mTOR signaling responses played important roles in MERS-CoV infection and the authors suggested this pathway as potential target [27]. In another very revealing study of patients with severe Influenza H1N1 pneumonia, early adjuvant treatment with corticosteroids and sirolimus was associated with improvement in clinical outcomes, such as hypoxia, multiple organ dysfunction, virus clearance, and shortened liberation of ventilator and ventilator and final activation of mTOR1 at the level of the lysosomal membrane. Another pathway of mTOR activation is also shown: Virions entering the eukaryotic cell using a membrane receptor (ACE-2) and activating MyD88 after lysosomal processing. In the end, mTOR1 works as a regulator via the phosphorylation of downstream effectors

days [61]. Of note, the mortality associated with Influenza pneumonia, as with SARS-CoV-2, is also attributed to acute respiratory distress syndrome (ARDS) [41]. Recent network-based drug repurposing models recently also identified mTOR inhibitors as potential useful drugs against SARS-CoV-2 [69]. Multiple ongoing prospective clinical trials are testing the pharmacological inhibition of mTOR against COVID-19 [11].

Another possible indirect link of the relationship between SARS-CoV-2 and the mTOR pathway is the relationship between COVID-19 and worse clinical outcomes in obese patients [17]. Obese patients suffer from "chronically overactivated" mTOR in multiple tissues [7]. The hypothesis that overactivated mTOR produce obesity was proved when S6K knockout mice showed resistance to obesity [54]. Obese patients infected with SARS-CoV-2 may overload an already hyperactivated mTOR system with the result of hyperproduction of virions and pro-inflammatory cytokines. Obese patients with COVID-19 may reach more easily the "set-point" or "threshold of tolerable inflammation" above which the cytokine storm syndrome is triggered (see Fig. 3). 


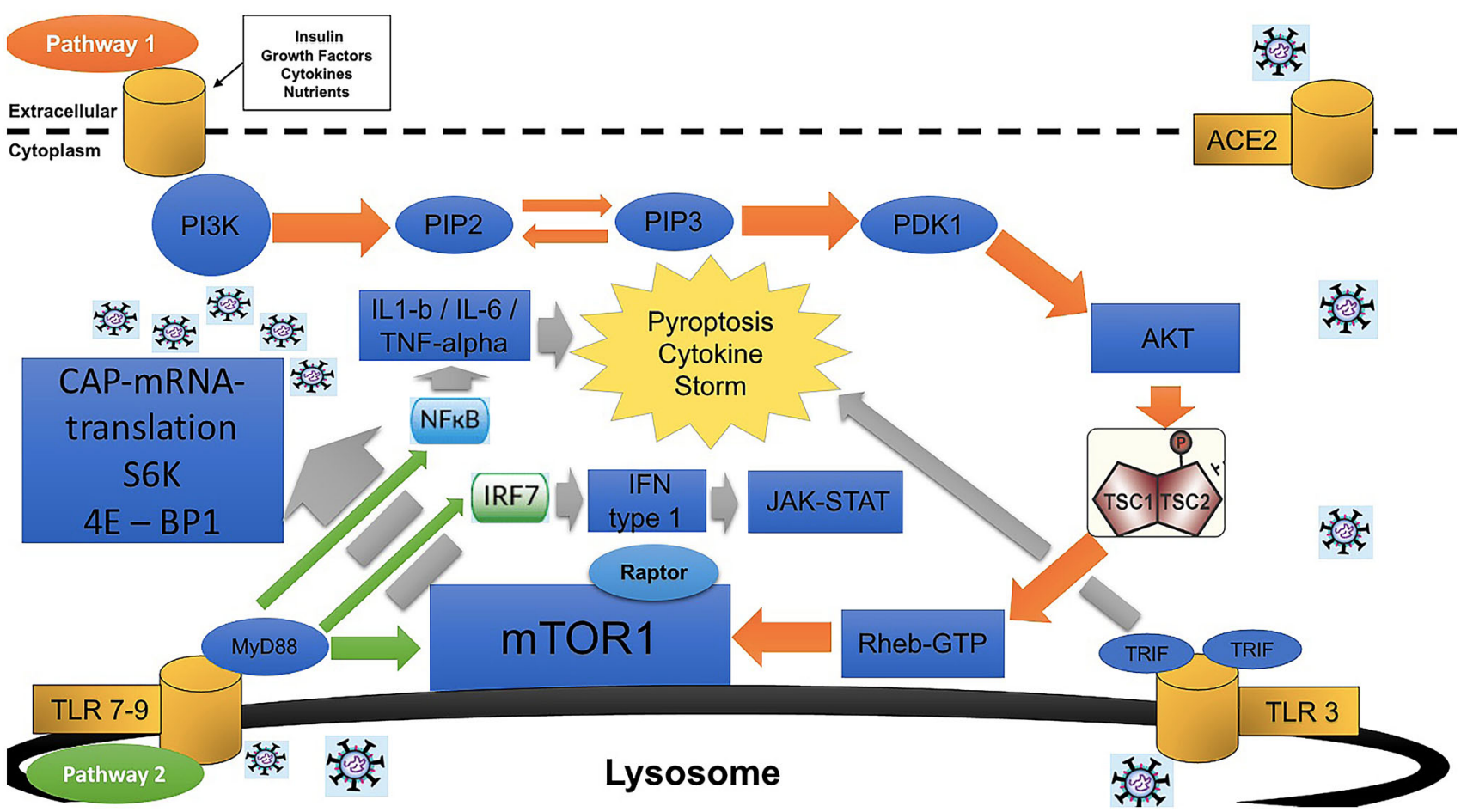

Fig. 2 A model of the integration of the activation of mTOR leading to the subsequent expression of the transcription factor NF-kb (IL-1b and IL-6) responsible of Inflammation and Cytokine Storm. The nutritional-hormonal pathway that begins with the activation of a transmembrane ligand receptor (Pathway 1 in orange arrows) and the processed viral-RNA pathway that begins at the lysosomal membrane with TLR7-9 activation (Pathway 2 in green arrows) both leading to mTOR activation and the downstream cascade in grey arrows

Fig. 3 Hypothesized model of "tolerable inflammation" threshold above which the cytokine storm syndrome might be triggered related to the potential mTOR activation and intermittent fasting (IF) role

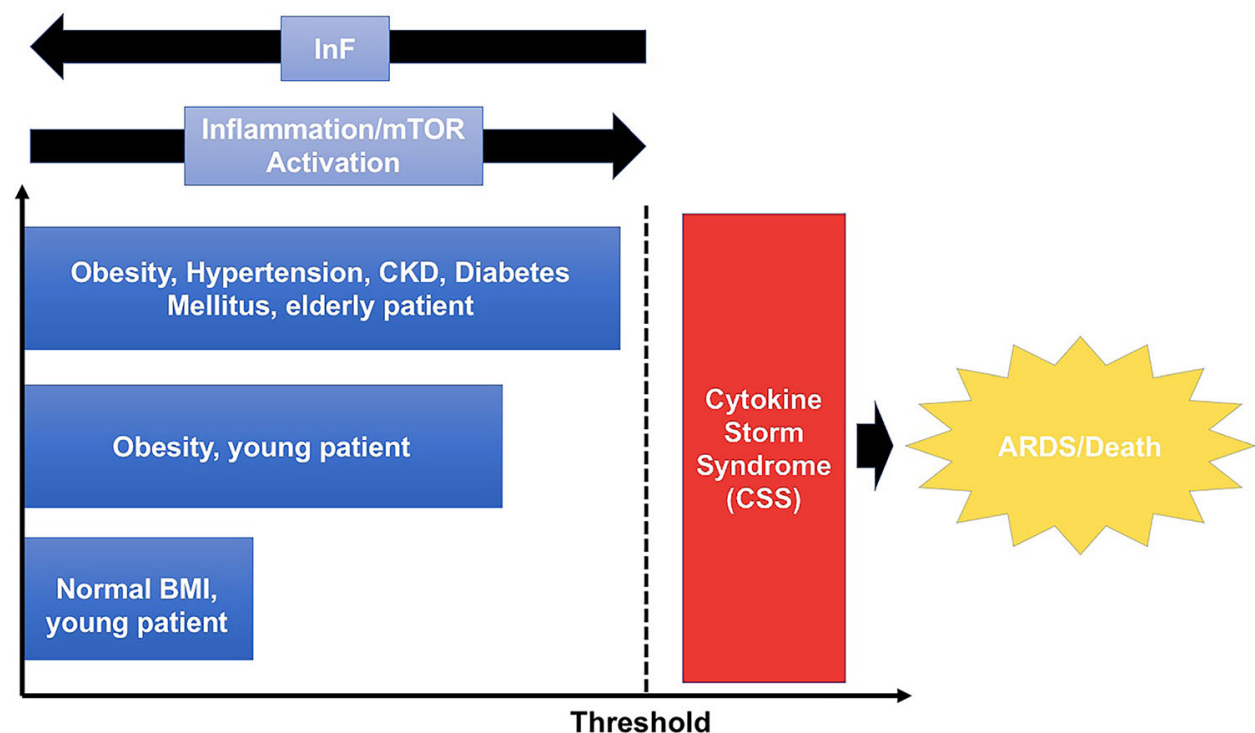

Based on the above, the possibility of alteration of the SARS-CoV-2 life cycle and its consequences through inhibition of mTOR and stimulation of autophagy with non-pharmacological (nutritional) strategies is intriguing.

\section{Intermittent fasting (IF)}

Recently there has been an increasing public and scientific interest about the possible health benefits of IF (mainly categorized as time restrictive feeding or alternate day 
fasting) beyond weight loss [1, 22, 23, 31, 35, 44]. Mammals have developed specific mechanisms over hundreds of thousands of years to overcome the lack of food and energy in the environment triggering stress signals. Of note, the same mechanisms have been seen in Nematodes and yeasts [31] which means that these ancient mechanisms have been highly conserved between species. These alternative metabolic pathways are extremely efficacious to handle cellular stress and starvation, keeping a high level of mental acuity and physical endurance among other health benefits including decreasing inflammation, promoting anti-aging effects and accelerating autophagy [36]. Multiple animal studies of IF have consistently proven to increase lifespan, decrease inflammation, treat diabetes and other metabolic diseases, improve cardiovascular health, and promote innumerable neurocognitive benefits (including neuro-protection against stroke) which has been reviewed in detail in previous reviews [31, 36]. This mechanism was probably not only created to use alternative sources of energy but also to clear cells from toxic molecules, R.O.S, DNA damage, cellular debris, and, probably, intracellular organisms through promotion of autophagy. It is well recognized that mTOR activation has "Pro-aging" effects and favors senescence later in life since it inhibits autophagy. mTOR is thought to be culprit of cellular accumulation of damaged organelles, oxidizedmisfolded proteins, and cellular debris since it will inhibit the clearance of those molecules with resultant "aging". In fact, the main mechanism of action of IF is the inhibition of mTOR and stimulation of autophagy.

\section{Intersection between COVID-19, IF, autophagy, and mTOR}

\section{Possible Direct anti-viral mechanisms of IF}

Inhibition of mTOR It was shown mainly in vitro that IF directly improves cellular stress adaptation (mainly against ROS generation), decrease inflammation and DNA damage, decrease mTOR expression [31], and promote autophagy [34] which is similar to the effects of Rapamycin in some animal models [19, 49, 63]. These effects could potentially be the link between the direct benefits of IF in COVID-19 due to the interruption of the SARS-CoV-2 viral cycle (decrease protein synthesis) with decrease in viral productivity and systemic inflammation.

In a "fed-state" the persistent activation of mTOR during COVID-19 through the TLR/MyD88 pathway and the nutritional-hormonal pathway may promote local and systemic inflammation (increase IL- $1 \mathrm{~b}$ and IL- 6 between others), with intracellular SARS-CoV-2 persistence due to increase of protein synthesis and replication. Viral persistence will be the link between immune-exhaustion, immune-senescence, and inflammation; with worse clinical outcomes in certain high-risk population [13]. In this scenario there will be a double-loop of positive feedback activation over mTOR (viral and nutritional-hormonal pathway) (see Fig. 2).

IF may not completely avoid the mucosal "Infection" of the host (since there may be some replication through the MyD88 pathway) but may prevent or ameliorate significantly the viral replication within the cell through inhibition of the nutritional-hormonal pathway (Pathway 1 on Fig. 2). Processed viral RNA in the lysosomal membrane during starvation may still stimulate some lysosomal TLR7-9 with subsequent activation of mTOR through the MyD88 pathway in Antigen Presenting Cells (APCs) with resulting activation of the transcription factor $\mathrm{NF}-\mathrm{kb}$ (Pathway 2 on Fig. 2) and IL-1b and IL-6 production but the volume of virion production may be less since one limb of the feedback loop (nutritional) will be absent. At this level the mTOR might remain transcriptionally active due to constant presence of processed viral RNA even during starvation but at an expected much lower level. The silencing of mTOR with IF may abolish in part some viral protein synthesis (if not the majority) since it will pause the 5'cap-dependent mRNA translation complex. The level of mTOR activation in the absence of activation of the nutritional-hormonal loop is difficult to predict but will need to be tested in experimental models. Recent publications encourage and promote the study of new strategies to promote autophagy in COVID-19 [9]. In the best clinical scenario, the contribution of the hormonal-nutritional pathway is of paramount importance for the viral cycle and in a starving mode the processed virions may not be enough to produce significant viral replication and inflammation. The clinical correlation of this possible findings remains to be defined and is poorly understood.

Promotion of autophagy The promotion of autophagy may accelerate the clearance of remaining and partially processed viral RNA near the lysosomal membrane. Evidence suggests that a specific form of autophagy, called "xenophagy", is responsible for the elimination of bacterial, viral or fungal pathogens [45]. Studies have also demonstrated that autophagy can attenuate lung inflammation when exposed to a pathogen [45] which may contribute to decrease the hyperinflammation associated with SARS-CoV-2. Autophagy is regulated by three protein complexes: ULK1 (ULK1, ATG13, RB1CC1/FIP200 and ATG101), class III PtdIns3K (ATG14, BECN1, PIK3R4/ VPS15 and PIK3C3/VPS34) and ATG16L1. mTOR is a direct inhibitor of ULK1. Under starvation conditions, MTOR is inactivated allowing ULK1 complex formation, and activation of the PtdIns $3 \mathrm{~K}$, which will result in the assemble of the autolysosome [5]. Cytoplasmic cargo 
includes damaged mitochondria, organelles, proteins, nucleic acids, intracellular bacteria, etc. Several different families of viruses, including coronavirus, have adapted by evolving a large variety of strategies to escape and/or to benefit via the inhibition and/or stimulation of autophagy at different stages of the process. It was recently reported that MERS-CoV infection actually blocks autophagy at the autolysosome formation stage via NSP6 and the accessory proteins $4 \mathrm{~b}$ and 5 which would prevent the late formation of mature autolysosomes (responsible for lysis of the cargo). The interplay between coronaviruses and autophagy is very complex and not completely understood. Multiple already approved drugs for other uses have shown promise in vitro on promoting autophagy and inhibiting some members of this family of viruses. It is being more recognized now that autophagy inducers generally will antagonize coronavirus replication. This are some examples [5]:

- Everolimus (Inhibition of MTOR): MERS-CoV inhibition

- Ivermectin (Inhibition of PAK1 and subsequent AKT phosphorylation): SARS-CoV-2 inhibition.

- Rapamycin/Sirolimus (Inhibition of MTOR): MERSCoV/MHV/TGEV/PEDV inhibition.

- Niclosamide (Inhibition of MTORC1 and ULK1 activities and induction of LC3B expression): MERS-CoV inhibition.

In a recent publication it was shown that SARS-CoV-2 inhibits autophagy through the downregulation of autophagy-inducing spermidine, and facilitates AKT1/SKP2dependent degradation of autophagy-initiating Beclin-1 (BECN1), which is reverted by Niclosamide [42]. In a recent genomic study of the SARS-CoV-2, it was found that the NSP6 protein of the virus binds with greater affinity to the endoplasmic reticulum (ER) which may allow the virus to inhibit autophagy, which prevents the degradation of viral particles by the final autolysosome [45]. It is believed that any therapeutic strategy that increases and enhance autophagy being pharmacological or nutritional may bring more benefits than harm.

\section{Role of IF in decreasing inflammation}

It is well-known that severe SARS-CoV patients has significantly higher serum levels of pro-inflammatory cytokines (IFN- $\gamma$, IL-1, IL-6, IL-12, and TGF $\beta$ ) and chemokines (CCL2, CXCL10, CXCL9, and IL-8) compared with SARS-CoV patients with mild disease or negative controls [10]. Interestingly, in very sick patients the anti-inflammatory cytokine IL-10 was decreased. The exacerbated immune response will drive mortality being the final events the CSS and ARDS once the threshold of inflammation is reached (see Fig. 3). IF is an attractive strategy to decrease lung and systemic inflammation. It is important to clarify that even though IF has shown much of its anti-inflammatory properties in animal studies COVID19 patients can suffer from such high systemic inflammatory levels above the mean that any change may correlate with improvement in clinical outcomes.

IF was shown to be a strong anti-inflammatory in multiple prior studies, mainly in animal studies and with conflicting results in humans $[24,31,35,36,58,60]$. In rats exposed to stroke experimental models IF decreased IL1-b, TNF-alpha, IL-6, and caused suppression of the "Inflammasome" [3]. Recently it was observed that IF regulates the NLRP3 Inflammasome activation through SIRT1 (deacetylases) having implications as a therapeutic target [58]. IF also resulted in reduced levels of mRNAs encoding the LPS receptor TLR4 and inducible nitric oxide synthase (iNOS) in the hippocampus of rats exposed to systemic LPS. Moreover, in another study IF prevented the LPSinduced elevation of IL- $1 \alpha$, IL- $1 b$, IFN- $\gamma$, RANTES, TNF- $\alpha$ and IL-6 [60]. A study in mice showed that enhancing mitochondrial energetics against damage-associated molecular patterns (DAMPs) may play a pivotal role in preventing inflammation during fasting and that it may be mediated, at least in part, through SIRT3-directed blunting of NLRP3 inflammasome assembly and activation [57]. These effects in animal studies were recently tested in a proof-of-concept study in pre-diabetes patients exposed to IF [53]. On this study IL-6, hs-CRP, and TNF-alpha did not change but IF did decrease markers of oxidative stress between other health benefits even though the number of patients were low $(n=8)$. If we consider inflammation a subtype of physiologic stress, IF could help to cope with it, triggering adaptive cellular responses, counteracting local diseases processes, decreasing DNA damage, stimulating local repair due to autophagy and increasing apoptosis of certain cells and cellular growth in others [31]. IF decreased markers of liver inflammation significantly in experimental rodent models fed with pro-inflammatory diets [35] possibly through an inhibition of TLR4, NF-kb, and interleukin signals [64]. A stressed Endoplasmic Reticulum (ER) is known to generate ROS which, in turn, activates the NLRP3 inflammasome and secretion of IL-1b. A recent study in rats showed also a potential therapeutic role of $\beta$-hydroxybutyrate (increased during IF) in suppressing the ER (stressed)-induced inflammasome activation [4]. This could have important implications in the context of SARS-CoV-2-stressed-ER due to the increased production of viral proteins. In another experimental model in rats with an experimentally induced stroke, IF could attenuate the inflammatory response and tissue damage by suppressing NLRP1 and NLRP3 inflammasome activity in neurons [16]. 
Recently was shown that isocaloric TRF (Time Restricted Feeding) during 8 weeks in males reduced many markers of inflammation such as tumor necrosis factor alpha, interleukin 6 , and interleukin $1 \mathrm{~b}$, and, increased adiponectin (an anti-inflammatory cytokine) [39]. It was proved that the Short Chain Fatty Acids (SCFAs) $\beta$-hydroxybutyrate (BHB) and acetoacetate, both elevated during starvation, inhibit the NLRP3 inflammasome. Of note, IF will increase the concentration of SCFAs significantly. SCFAs can inhibit the inflammasome by themselves, probably due to its high volume of distribution and high cell membrane penetration. SCFAs were shown to reduce the NLRP3 inflammasome-mediated interleukin (IL)-1 $\beta$ and IL-18 production in human monocytes [66] which will be extremely important in the context of SARS-CoV-2induced-CSS.

Studies of IF on patients with chronic inflammatory diseases can bring light to the possible benefits against COVID-19. A very revealing study showed that patients with Rheumatoid Arthritis (RA) had significant clinical improvement (pain and inflammation) after a period of fasting if a vegetarian diet was followed thereafter [40]. Another study in overweight Asthmatic female patients exposed to IF showed a significant decrease in the levels of TNF-alpha and markers or oxidative stress (8-isoprostane, nitro-tyrosine, protein carbonyls, and 4-hydroxynonenal adducts) with improved clinical response. It showed that prolonged fasting blunted the NLRP3 inflammasome and Th2 cell activation in steroid-naive asthmatics as well as diminished the airway epithelial cell cytokine production [20]. The last two studies show a clear benefit of IF on two very common systemic inflammatory diseases in humans (Rheumatoid arthritis and asthma) which could be applied to COVID-19. The relationship between nutrition and inflammation also can be attenuated by IF in COVID-19 patients. A diet rich in carbs and fat increases the levels of insulin and leptin. Leptin usually reflects a pro-inflammatory state whereas adiponectin and ghrelin (both increased while fasting) can suppress inflammation and increase insulin sensitivity. Adiponectin also possess antiatherosclerotic and anti-inflammatory effects by inhibiting the adhesion of monocytes to endothelial cells [33]. Importantly, it has been seen that IF reverses the metabolic syndrome in rodents in experimental models [31] which could have important clinical implications since obesity is a well-known risk factor for worse clinical outcomes in COVID-19. Of note even weight loss may be seen as only one of the positive outcomes of IF, in the case of COVI-19 it could have a direct impact on mortality and morbidity.
Decrease local gastrointestinal inflammation and repair of the gastrointestinal epithelial lining

It is well known that SARS-CoV-2 has receptors in the gastrointestinal epithelial cells and can present clinically with GI symptoms [56]. To have a structural intact and functional GI epithelial barrier must be extremely important to prevent infection of epithelial cells with SARS$\mathrm{CoV}-2$. In fact, the GI tract may represent a reservoir for SARS-CoV-2 even after decolonization of the nasopharynx and respiratory mucosa. Even in the absence of gastrointestinal symptoms, there may still be local inflammation, immune activation, and, in some cases, a "leaky gut" with microbial translocation, especially in obese patients [59]. The significant increase of Short Chain Fatty Acids seen during IF will decrease the local gut inflammation and may contribute to reverse the damage to the epithelial tight junctions. The concept of enhancement of Pluripotential Stem cells (PSCs) with inhibition of mTOR during fasted states has been described before [65]. In fact it has been shown that IF promotes hematopoietic stem cell activation and regeneration of immune cells [8]. Interestingly, recently was shown that Paneth cells in the crypts of the gut mucosa, may promote the growth of Intestinal Stem Cells (ISCs) during fasting through inhibition of mTOR, result that was also reproduced using Rapamycin. That could mean that IF may have implications for epithelial and tight junction repair after SARS-CoV-2 initial damage since Paneth cells may sense the lack of nutrients as a signal of cellular stress. This study showed for the first time a link between the host nutritional status and stem cell function through mTOR inhibition since mTOR controls ISCs function depending on nutritional factors [2]. IF also showed to increase ISCs numbers, increase ISCs self-renewal, increase ISCs regeneration, and decrease ISCs differentiation [2]. Inhibition of mTOR, Insulin, and IGF1during IF may promote the growth and self-renewal of ISCs with repair of the epithelial damage. It is clear that IF shall have benefits in the gut hemostasis since it has been showed to limit the intestinal toxicity during chemotherapy probably reflecting the increase activity of ISCs [2].

A potential change in the composition of the microbiome to a less-inflammatory biotype (phenotype switch to the Firmicutes and Lactobacillus with detrimental effects on Proteobacterias and Prevotella) with IF may have also important implications in mounting an appropriate immune response against SARS-CoV-2 and less systemic inflammation. 


\section{Potential clinical implications}

Clinical benefits of intermittent fasting are still under investigation and multiple studies are showing promising results towards rheumatic diseases, neoplasms, diabetes, metabolic syndrome, cognitive dysfunction and mental health $[32,38]$.

COVID-19 tends to present with a severe pulmonary compromise in patients with chronic metabolic conditions such as diabetes, obesity, metabolic syndrome and cardiovascular diseases [28, 67]. In that sense, some studies have found that higher glucose levels at admission were linked to mortality and worse clinical outcomes in patients with COVID-19 [62]. Intermittent fasting could play a role in preventing adverse COVID-19 outcomes among obese and diabetic patients. Recent investigations have strongly suggested a relationship between a well-designed intermittent fasting schedule and a considerable improvement in glycemic control along with beneficial metabolic effects $[6,18]$. On another aspect, some studies have reported a potential increase in vital lung capacity and mean/peak expiratory flow among patients during Ramadan fasting period compared to post-Ramadan [25]. The potential clinical implication of this findings could lie in the importance of IF in preserving an adequate baseline lung health, that would allow a lower degree of pulmonary compromise during SARS-CoV-2 infection.

Other investigations are postulating a strong role of IF in the reduction of cardiovascular risk, since this intervention have shown to lower blood pressure through the parasympathetic system, reduce blood cholesterol levels and directly inhibit the development of atherosclerotic plaques due to its intrinsic immunomodulatory role [15]. Cardiovascular diseases have shown to carry a higher risk of SARS-CoV-2 infection and a higher risk of progression to pneumonia and the need for ICU care [30]. This may be explained by SARS-CoV-2 infection acting as a precipitating factor that may worsen a previously diminished cardiac functional reserve owing to myocardial ischemia or myocarditis [67]. IF may play a role in diminishing this burden of cardiovascular disease and as an effective nonpharmacological preventive method.

Time-restricted feeding (TRF) has been described with different schedules ranging from a 16 -h fasts $/ 8 \mathrm{~h}$ feeding times, 18 -h fasts $/ 6 \mathrm{~h}$ feeding times and 20 - $\mathrm{h}$ fasts/4-h feed times [14]. We consider that the optimal goal should aim to increase periodically the hours of fasting and try to reach the hypothetical goal of one meal a day (OMD) as tolerated by the individual. In that sense, further studies should consider the importance of an ideal timeframe for the fasting period as well as the optimal time of day when it occurs.
Weight loss with decrease of the BMI, improvement of cardiovascular disease and metabolic syndrome, improvement of diabetes mellitus, decrease of systemic inflammation, inhibition of mTOR with control of viral replication, and promotion of autophagy could be highly appreciated therapeutic effects from an intervention that has a very low cost and with no mayor risks.

\section{Conclusion}

The COVID-19 pandemic is still a public health concern worldwide. Molecular theoretical models show potential beneficial effects of IF on the interruption of SARS-CoV-2 life cycle through inhibition of mTOR and promotion of autophagy. Studies are needed in order to evaluate different intermittent fasting (IF) regimens and the clinical implications on the prevention and treatment of COVID-19 in humans. Furthermore, special populations at risk could benefit from these cost-effective preventive interventions that may develop into policies and public health strategies.

Acknowledgements We thank Dr. Pascual Chiarella for his support during the final review of the manuscript.

\section{Declaration}

Conflict of interest The authors declare that they have no conflict of interest.

\section{References}

1. Alam I, Gul R, Chong J, Tan CTY, Chin HX, Wong G, et al. Recurrent circadian fasting (RCF) improves blood pressure, biomarkers of cardiometabolic risk and regulates inflammation in men. J Transl Med. 2019;17(1):272.

2. Alonso S, Yilmaz OH. Nutritional regulation of intestinal stem cells. Annu Rev Nutr. 2018;38:273-301.

3. Arumugam TV, Phillips TM, Cheng A, Morrell CH, Mattson MP, Wan R. Age and energy intake interact to modify cell stress pathways and stroke outcome. Ann Neurol. 2010;67(1):41-52.

4. Bae HR, Kim DH, Park MH, Lee B, Kim MJ, Lee EK, et al. betaHydroxybutyrate suppresses inflammasome formation by ameliorating endoplasmic reticulum stress via AMPK activation. Oncotarget. 2016;7(41):66444-54.

5. Bello-Perez M, Sola I, Novoa B, Klionsky DJ, Falco A. Canonical and noncanonical autophagy as potential targets for COVID19. Cells. 2020;9(7):1619.

6. Bener A, Al-Hamaq AAOA, Öztürk M, et al. Effect of ramadan fasting on glycemic control and other essential variables in diabetic patients. Ann Afr Med. 2018;17(4):196-202. https://doi.org/ 10.4103/aam.aam_63_17.

7. Bolourian A, Mojtahedi Z. Obesity and COVID-19: the mTOR pathway as a possible culprit. Obes Rev. 2020;21(9):e13084.

8. Buono R, Longo VD. When fasting gets tough, the tough immune cells get going-or die. Cell. 2019;178(5):1038-40. 
9. Calender A, Israel-Biet D, Valeyre D, Pacheco Y. Modeling potential autophagy pathways in COVID-19 and sarcoidosis. Trends Immunol. 2020;41(10):856-9.

10. Channappanavar R, Perlman S. Pathogenic human coronavirus infections: causes and consequences of cytokine storm and immunopathology. Semin Immunopathol. 2017;39(5):529-39.

11. ClinicalTrials.gov. [Internet]. Bethesda (MD): National Library of Medicine (US), National Center for Biomedical Information. [updated $2021 \mathrm{Jan}$ ]. Available from: https://clinicaltrials.gov/ct2/ results? cond $=$ Covid $19 \&$ term $=$ sirolimus $\&$ cntry $=\&$ state $=\&$ city $=$ $\&$ dist $=$. [Accessed 12 January 2021].

12. Cooray S. The pivotal role of phosphatidylinositol 3-kinase-Akt signal transduction in virus survival. J Gen Virol. 2004;85(Pt 5):1065-76.

13. Cunha LL, Perazzio SF, Azzi J, Cravedi P, Riella LV. Remodeling of the immune response with aging: immunosenescence and its potential impact on COVID-19 immune response. Front Immunol. 2020;11:1748.

14. Dong TA, Sandesara PB, Dhindsa DS, et al. Intermittent fasting: a heart healthy dietary pattern? Am J Med. 2020;133(8):901-7. https://doi.org/10.1016/j.amjmed.2020.03.030.

15. Dong TA, Sandesara PB, Dhindsa DS, et al. Intermittent fasting: a heart healthy dietary pattern? Am J Med. 2020;133(8):901-7. https://doi.org/10.1016/j.amjmed.2020.03.030.

16. Fann DY, Santro T, Manzanero S, Widiapradja A, Cheng YL, Lee SY, et al. Intermittent fasting attenuates inflammasome activity in ischemic stroke. Exp Neurol. 2014;257:114-9.

17. Finer N, Garnett SP, Bruun JM. COVID-19 and obesity. Clin Obes. 2020;10(3):e12365.

18. Ganesan K, Habboush Y, Dagogo-Jack S. Calorie restriction and intermittent fasting: impact on glycemic control in people with diabetes. Diabetes Spectr. 2020;33(2):143-8. https://doi.org/10. 2337/ds19-0064.

19. Gao S, Liu W, Zhuo X, Wang L, Wang G, Sun T, et al. The activation of mTOR is required for monocyte pro-inflammatory response in patients with coronary artery disease. Clin Sci (Lond). 2015;128(8):517-26.

20. Han K, Nguyen A, Traba J, Yao X, Kaler M, Huffstutler RD, et al. A Pilot Study To Investigate the Immune-Modulatory Effects of Fasting in Steroid-Naive Mild Asthmatics. J Immunol (Baltimore, Md, 1950). 2018;201(5):1382-8.

21. Harrison AG, Lin T, Wang P. Mechanisms of SARS-CoV-2 transmission and pathogenesis. Trends Immunol. 2020;41(12):1100-15. https://doi.org/10.1016/j.it.2020.10.004.

22. Harvie MN, Howell T. Could intermittent energy restriction and intermittent fasting reduce rates of cancer in obese, overweight, and normal-weight subjects? a summary of evidence. Adv Nutr. 2016;7(4):690-705.

23. Harvie MN, Pegington M, Mattson MP, Frystyk J, Dillon B, Evans G, et al. The effects of intermittent or continuous energy restriction on weight loss and metabolic disease risk markers: a randomized trial in young overweight women. Int J Obes (Lond). 2011;35(5):714-27.

24. Herieka M, Erridge C. High-fat meal induced postprandial inflammation. Mol Nutr Food Res. 2014;58(1):136-46.

25. Javanmard SH, Otroj Z. Ramadan fasting and risk of Covid-19. Int J Prev Med. 2020;11:60. https://doi.org/10.4103/ijpvm. IJPVM_236_20.

26. Kader M, Alaoui-El-Azher M, Vorhauer J, Kode BB, Wells JZ, Stolz D, et al. MyD88-dependent inflammasome activation and autophagy inhibition contributes to Ehrlichia-induced liver injury and toxic shock. PLoS Pathog. 2017;13(10):e1006644.

27. Kindrachuk J, Ork B, Hart BJ, Mazur S, Holbrook MR, Frieman $\mathrm{MB}$, et al. Antiviral potential of ERK/MAPK and PI3K/AKT/ mTOR signaling modulation for middle east respiratory syndrome coronavirus infection as identified by temporal kinome analysis. Antimicrob Agents Chemother. 2015;59(2):1088-99.

28. Korakas E, Ikonomidis I, Kousathana F, et al. Obesity and COVID-19: immune and metabolic derangement as a possible link to adverse clinical outcomes. Am J Physiol Endocrinol Metab. 2020;319(1):E105-9. https://doi.org/10.1152/ajpendo. 00198.2020.

29. Le Sage V, Cinti A, Amorim R, Mouland AJ. Adapting the stress response: viral subversion of the mTOR signaling pathway. Viruses. 2016;8(6):152.

30. Li B, Yang J, Zhao F, et al. Prevalence and impact of cardiovascular metabolic diseases on COVID-19 in China. Clin Res Cardiol. 2020;109(5):531-8. https://doi.org/10.1007/s00392-02001626-9.

31. Longo VD, Mattson MP. Fasting: molecular mechanisms and clinical applications. Cell Metab. 2014;19(2):181-92.

32. Longo VD, Panda S. Fasting, circadian rhythms, and time-restricted feeding in healthy lifespan. Cell Metab. 2016;23(6):1048-59. https://doi.org/10.1016/j.cmet.2016.06.001.

33. Malinowski B, Zalewska K, Wesierska A, Sokolowska MM, Socha M, Liczner G, et al. Intermittent fasting in cardiovascular disorders-an overview. Nutrients. 2019;11(3):673.

34. Mani K, Javaheri A, Diwan A. Lysosomes mediate benefits of intermittent fasting in cardiometabolic disease: the janitor is the undercover boss. Compr Physiol. 2018;8(4):1639-67.

35. Marinho TS, Ornellas F, Barbosa-da-Silva S, Mandarim-deLacerda CA, Aguila MB. Beneficial effects of intermittent fasting on steatosis and inflammation of the liver in mice fed a high-fat or a high-fructose diet. Nutrition. 2019;65:103-12.

36. Mattson MP, Longo VD, Harvie M. Impact of intermittent fasting on health and disease processes. Ageing Res Rev. 2017;39:46-58.

37. Mellet J, Pepper MS. A COVID-19 vaccine: big strides come with big challenges. Vaccines (Basel). 2021;9(1):E39. https://doi. org/10.3390/vaccines 9010039 .

38. Mindikoglu AL, Abdulsada MM, Jain A, et al. Intermittent fasting from dawn to sunset for 30 consecutive days is associated with anticancer proteomic signature and upregulates key regulatory proteins of glucose and lipid metabolism, circadian clock, DNA repair, cytoskeleton remodeling, immune system and cognitive function in healthy subjects. $\mathrm{J}$ Proteomics. 2020;217:103645. https://doi.org/10.1016/j.jprot.2020.103645.

39. Moro T, Tinsley G, Bianco A, Marcolin G, Pacelli QF, Battaglia $\mathrm{G}$, et al. Effects of eight weeks of time-restricted feeding (16/8) on basal metabolism, maximal strength, body composition, inflammation, and cardiovascular risk factors in resistancetrained males. J Transl Med. 2016;14(1):290.

40. Muller H, de Toledo FW, Resch KL. Fasting followed by vegetarian diet in patients with rheumatoid arthritis: a systematic review. Scand J Rheumatol. 2001;30(1):1-10.

41. Ng HH, Narasaraju T, Phoon MC, Sim MK, Seet JE, Chow VT. Doxycycline treatment attenuates acute lung injury in mice infected with virulent influenza H3N2 virus: involvement of matrix metalloproteinases. Exp Mol Pathol. 2012;92(3):287-95.

42. Nils C, Gassen JP, Bajaj T, Dethloff F, Emanuel J, Weckmann K, et al. Analysis of SARS-CoV-2-controlled autophagy reveals spermidine, MK-2206, and niclosamide as putative antiviral therapeutics. 2020. bioRxiv. https://doi.org/10.1101/2020.04.15. 997254

43. Nouwen LV, Everts B. Pathogens MenTORing macrophages and dendritic cells: manipulation of mTOR and cellular metabolism to promote immune escape. Cells. 2020;9(1):161.

44. Patterson RE, Sears DD. Metabolic effects of intermittent fasting. Annu Rev Nutr. 2017;37:371-93.

45. Pehote G, Vij N. Autophagy Augmentation to Alleviate Immune Response Dysfunction, and Resolve Respiratory and COVID-19 Exacerbations. Cells. 2020;9(9):1952. 
46. Ramaiah MJ. mTOR inhibition and p53 activation, microRNAs: the possible therapy against pandemic COVID-19. Gene Rep. 2020;20:100765.

47. 47. RECOVERY Collaborative Group, Horby P, Lim WS, et al. Dexamethasone in Hospitalized Patients with Covid-19. N Engl J Med. 2021;384(8):693-704. https://doi.org/10.1056/ NEJMoa2021436

48. Rothan HA, Byrareddy SN. The epidemiology and pathogenesis of coronavirus disease (COVID-19) outbreak. J Autoimmun. 2020;109:102433.

49. Schmeisser K, Parker JA. Pleiotropic effects of mTOR and autophagy during development and aging. Front Cell Dev Biol. 2019;7:192.

50. Shin YK, Liu Q, Tikoo SK, Babiuk LA, Zhou Y. Effect of the phosphatidylinositol 3-kinase/Akt pathway on influenza A virus propagation. J Gen Virol. 2007;88(Pt 3):942-50.

51. Sreepadmanabh M, Sahu AK, Chande A. COVID-19: advances in diagnostic tools, treatment strategies, and vaccine development. J Biosci. 2020;45(1):148. https://doi.org/10.1007/s12038-02000114-6.

52. Stöhr S, Costa R, Sandmann L, Westhaus S, Pfaender S, et al. Host cell mTORC1 is required for HCV RNA replication. Gut. 2016;65(12):2017-28.

53. Sutton EF, Beyl R, Early KS, Cefalu WT, Ravussin E, Peterson CM. Early time-restricted feeding improves insulin sensitivity, blood pressure, and oxidative stress even without weight loss in men with prediabetes. Cell Metab. 2018;27(6):1212-21.e3.

54. Tavares MR, Pavan IC, Amaral CL, Meneguello L, Luchessi AD, Simabuco FM. The S6K protein family in health and disease. Life Sci. 2015;131:1-10.

55. Terrazzano G, Rubino V, Palatucci AT, Giovazzino A, Carriero F, Ruggiero G. An open question: is it rational to inhibit the mTor-dependent pathway as COVID-19 therapy? Front Pharmacol. 2020;11:856.

56. Tian Y, Rong L, Nian W, He Y. Review article: gastrointestinal features in COVID-19 and the possibility of faecal transmission. Aliment Pharmacol Ther. 2020;51(9):843-51.

57. Traba J, Geiger SS, Kwarteng-Siaw M, Han K, Ra OH, Siegel RM, et al. Prolonged fasting suppresses mitochondrial NLRP3 inflammasome assembly and activation via SIRT3-mediated activation of superoxide dismutase 2. J Biol Chem. 2017;292(29):12153-64.

58. Traba J, Kwarteng-Siaw M, Okoli TC, Li J, Huffstutler RD, Bray $A$, et al. Fasting and refeeding differentially regulate NLRP3 inflammasome activation in human subjects. J Clin Investig. 2015;125(12):4592-600.

59. Uzzan M, Corcos O, Martin JC, Treton X, Bouhnik Y. Why is SARS-CoV-2 infection more severe in obese men? the gut
lymphatics—Lung axis hypothesis. Med Hypotheses. 2020;144:110023.

60. Vasconcelos AR, Yshii LM, Viel TA, Buck HS, Mattson MP, Scavone C, et al. Intermittent fasting attenuates lipopolysaccharide-induced neuroinflammation and memory impairment. J Neuroinflammation. 2014;11:85.

61. Wang CH, Chung FT, Lin SM, Huang SY, Chou CL, Lee KY, et al. Adjuvant treatment with a mammalian target of rapamycin inhibitor, sirolimus, and steroids improves outcomes in patients with severe H1N1 pneumonia and acute respiratory failure. Crit Care Med. 2014;42(2):313-21.

62. Wang S, Ma P, Zhang S, et al. Fasting blood glucose at admission is an independent predictor for 28-day mortality in patients with COVID-19 without previous diagnosis of diabetes: a multi-centre retrospective study. Diabetologia. 2020;63(10):2102-11. https:// doi.org/10.1007/s00125-020-05209-1.

63. Wijngaarden MA, Bakker LE, van der Zon GC, t Hoen PA, van Dijk KW, Jazet IM, et al. Regulation of skeletal muscle energy/ nutrient-sensing pathways during metabolic adaptation to fasting in healthy humans. Am J Physiol Endocrinol Metab. 2014;307(10):E885-95.

64. Yang W, Cao M, Mao X, Wei X, Li X, Chen G, et al. Alternateday fasting protects the livers of mice against high-fat diet-induced inflammation associated with the suppression of toll-like receptor 4/nuclear factor kappaB signaling. Nutr Res (New York, NY). 2016;36(6):586-93.

65. Yilmaz OH, Katajisto P, Lamming DW, Gultekin Y, Bauer-Rowe KE, Sengupta S, et al. mTORC1 in the Paneth cell niche couples intestinal stem-cell function to calorie intake. Nature. 2012;486(7404):490-5.

66. Youm YH, Nguyen KY, Grant RW, Goldberg EL, Bodogai M, Kim D, et al. The ketone metabolite beta-hydroxybutyrate blocks NLRP3 inflammasome-mediated inflammatory disease. Nat Med. 2015;21(3):263-9.

67. Zheng YY, Ma YT, Zhang JY, Xie X. COVID-19 and the cardiovascular system. Nat Rev Cardiol. 2020;17(5):259-60. https:// doi.org/10.1038/s41569-020-0360-5.

68. Zheng Y, Li R, Liu S. Immunoregulation with mTOR inhibitors to prevent COVID-19 severity: A novel intervention strategy beyond vaccines and specific antiviral medicines. J Med Virol. 2020;92(9):1495-1500. https://doi.org/10.1002/jmv.26009

69. Zhou Y, Hou Y, Shen J, Huang Y, Martin W, Cheng F. Networkbased drug repurposing for novel coronavirus 2019-nCoV/SARSCoV-2. Cell Discov. 2020;6:14.

Publisher's Note Springer Nature remains neutral with regard to jurisdictional claims in published maps and institutional affiliations. 